\title{
Lattice Boltzmann method for compressible flows with high Mach numbers
}

\author{
Huidan $\mathrm{Yu}^{1,2,3, *}$ and Kaihua Zhao ${ }^{1}$ \\ ${ }^{1}$ Department of Physics and Center for Nonlinear Sciences, Peking University, Beijing 100871, China \\ ${ }^{2}$ Laboratory of Nonlinear Mechanics, Institute of Mechanics, Chinese Academy of Science, Beijing 100800, China \\ ${ }^{3}$ Department of Physics, Zhejiang Normal University, Jinhua 321004, Zhejiang, China
}

(Received 28 September 1999)

\begin{abstract}
In this paper we present a lattice Boltzmann model to simulate compressible flows by introducing an attractive force. This scheme has two main advantages: one is to soften sound speed effectively, which greatly raises the Mach number (up to 5); another is its relative simple procedure. Simulations of the March cone and the comparison between theoretical expectations and simulations demonstrate that the scheme is effective in the simulation of compressible flows with high Mach numbers, which would create many new applications.
\end{abstract}

PACS number(s): 51.10. $+\mathrm{y}, 47.40 . \mathrm{Nm}, 02.70 .-\mathrm{c}$

\section{INTRODUCTION}

In 1986, Frisch, Hasslacher, and Pomeau [1] proposed the first lattice gas model for the two-dimensional incompressible Navier-Stokes equation. Since then, much attention has been paid to this relatively new method [2]. Many theoretical and numerical studies have been concentrated on a variety of physics phenomena from shock formation [3] to flows in porous media [4], magnetohydrodynamics [5], phase separation [6,7], and turbulence [8]. Recently, the lattice Boltzmann (LB) method originated from lattice gas automata has been developed to be an alternative and promising numerical scheme for simulating fluid flow and modeling physics in fluids. The scheme is particularly successful in the applications involving interfacial dynamics and complex boundaries, especially when the effects of compressibility can be neglected. However, there exist a number of situations where such approximation is inappropriate $[9,10]$. Although several analytical and numerical LB techniques have been developed to treat compressible flows, no solutions are satisfactory.

In 1992, Alexander et al. [11] presented a selectable sound speed model to simulate compressible flow by selecting the parameters of the equilibrium distribution appropriately to set the sound speed as low as possible. In their model, the following single-component LB equation with a Bhatnagar-Gross-Krook (BGK) collision term describes the evolution of the distribution function $f_{a}(\vec{x}, t)$ in space $\vec{x}$ and time $\mathrm{t}$,

$$
f_{a}\left(\vec{x}+\vec{e}_{a}, t+1\right)=f_{a}(\vec{x}, t)+\Omega_{a},
$$

$$
a=1, \ldots, b,
$$

where $\Omega_{a}$ is the collision operator,

$$
\Omega_{a}=-\frac{1}{\tau}\left[f_{a}(\vec{x}, t)-f_{a}^{e q}(\vec{x}, t)\right]
$$

with $\tau$ the single relaxation time scale for approach to the desired equilibrium distribution. The set of vectors $\left\{\vec{e}_{a} ; a\right.$ $=0, \ldots, b\}$ are the possible velocities a particle can have in order to move from a lattice site to one of the $b$ numbers of the nearest-neighboring sites at each time step. $\vec{e}_{a}=0$ ( $a$ $=0)$ is associated with the rest ("stopped") particles, and $\left|\vec{e}_{a}\right|=c,(a=1, \ldots, b)$ where $c$ is the lattice constant. The macroscopic number density $\rho(\vec{x}, t)$ and velocity $\vec{u}(\vec{x}, t)$ of the fluid are obtained from $f_{a}$ as $\rho=\Sigma_{a} f_{a}$ and $\rho \vec{u}$ $=\Sigma_{a} f_{a} \vec{e}_{a}$. Equations (1) and (2) represent the relation of the distribution function to its equilibrium value, $f_{a}^{e q}$, which is a function of $\rho$ and $\vec{u}$ only. The choice of $f_{a}^{e q}$ has to ensure that the macroscopic fluid equation obtained from Eq. (1) by the Chapman-Enskog expansion agrees with the Navier-Stokes equations. The functional form of $f_{a}^{e q}$ depends on the structure of the lattice and is usually not uniquely determined.

For an FHP7-bit model (i.e., $b=6, c=1$ ), the form of $f_{a}^{e q}$ is usually shown as follows:

$$
f_{a}^{e q}=\frac{\rho}{3}\left[c_{s}^{2}+\vec{e}_{a} \cdot \vec{u}+2\left(\vec{e}_{a} \cdot \vec{u}\right)^{2}-\frac{1}{2} u^{2}\right],
$$

TABLE I. Effective sound speed comparison at different $g$ when $c_{s}=0.52, \tau=1, A=0.1$.

\begin{tabular}{lllllllllll}
\hline \hline$g$ & 0.90 & 0.91 & 0.92 & 0.93 & 0.94 & 0.95 & 0.96 & 0.97 & 0.98 & 0.99 \\
$T$ & 426 & 450 & 476 & 510 & 550 & 602 & 673 & 777 & 952 & 1350 \\
$c_{s}^{*}$ & 0.164 & 0.156 & 0.147 & 0.138 & 0.127 & 0.116 & 0.104 & 0.0901 & 0.074 & 0.052 \\
$c_{s}^{\prime}$ & 0.164 & 0.156 & 0.147 & 0.137 & 0.127 & 0.120 & 0.106 & 0.0964 & 0.0767 & 0.0556 \\
\hline \hline
\end{tabular}

\footnotetext{
*Electronic address: yu@jazz.me.psu.edu
} 
TABLE II. Effective sound speed comparison at different $c_{s}$ when $g=0.91, \tau=1, A=0.1, T=450$.

\begin{tabular}{llllllllll}
\hline$c_{s}$ & 0.48 & 0.50 & 0.52 & 0.54 & 0.56 & 0.58 & 0.60 & 0.62 & 0.64
\end{tabular}

$\begin{array}{lllllllllll}c_{s}^{*} & 0.144 & 0.150 & 0.156 & 0.162 & 0.168 & 0.174 & 0.180 & 0.186 & 0.192\end{array}$

$\begin{array}{lllllllllll}c_{s}^{\prime} & 0.144 & 0.149 & 0.156 & 0.162 & 0.167 & 0.171 & 0.176 & 0.182 & 0.189\end{array}$

$$
f_{0}^{e q}=\rho\left[1-2 c_{s}^{2}-u^{2}\right] .
$$

The corresponding macroscopic dynamic equation is

$$
\rho\left[\partial_{t} \vec{u}+(\vec{u} \cdot \nabla) \vec{u}\right]=-c_{s}^{2} \nabla \rho+\nabla^{2}(\eta \vec{u})+\nabla[\nabla \cdot(\zeta \vec{u})],
$$

where

$$
\eta=\frac{2 \tau-1}{8} \rho, \quad \zeta=\frac{2 \tau-1}{4}\left(1-2 c_{s}^{2}\right) \rho
$$

are the kinetic viscosity and the bulk viscosity, respectively.

As Alexander et al. [11,12] demonstrated, the main advantage of their model is that it has a selectable sound speed. In order to simulate compressible flows with high Mach numbers, sound speed must be selected as small as possible. However, the non-negativeness requirement of the equilibrium distribution functions limits such selection to a very narrow range. As a result, they still cannot simulate compressible flows with a high Mach number. Another LB model for compressible fluids [13] (called the Lattice BGK model) introduced a nonlinear derivation from the NavierStokes equation, but unfortunately it also has a limitation to raise the Mach number. There also exist two other models $[14,15]$ but they are so complicated that the advantages of the LB method are lost.

We found that it is crucial to avoid the distribution functions being negative when we construct new LB models. Our model below did satisfy this requirement.

\section{LB MODEL FOR COMPRESSIBLE FLOWS} force

In order to soften sound speed, we introduce an attractive

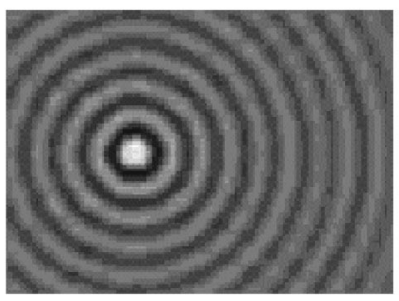

$$
\Pi^{3}=0
$$

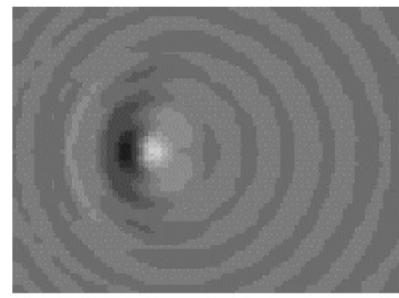

$\mathbb{M}^{3}=0.49$

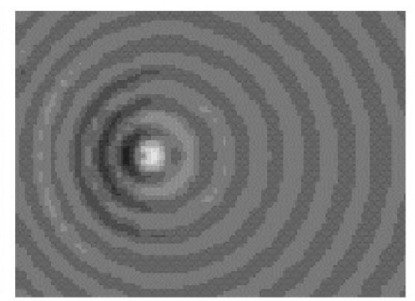

$$
\mu^{3}=0.29
$$

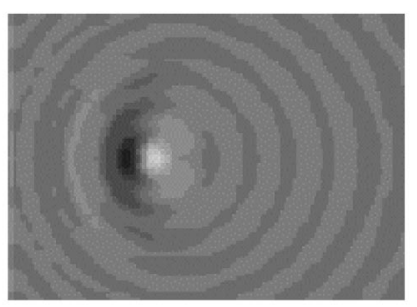

$M^{3}=0.68$

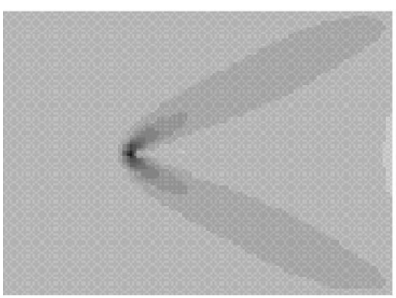

$m^{\prime}=2.16$

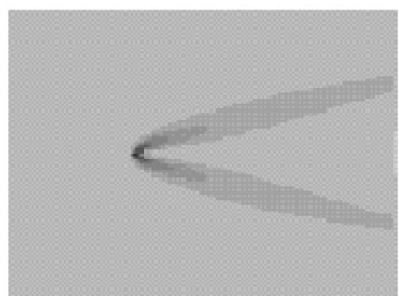

$\mathrm{m}^{\prime}=4.3$

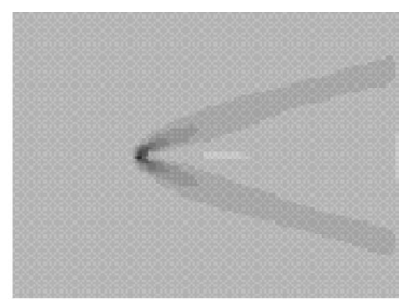

$m^{\prime}=3.23$

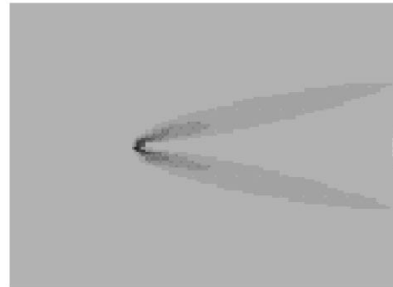

$\mathrm{m}^{\prime}=5.03$
FIG. 2. Mach cone simulation at different Mach numbers.

$$
\begin{aligned}
\Phi_{a}= & \frac{4}{9} g c_{s}^{2}\left\{\rho\left(\vec{x}+\vec{e}_{a}\right)-\rho\left(\vec{x}-\vec{e}_{a}\right)\right. \\
& \left.-\frac{1}{8}\left[\rho\left(\vec{x}+2 \vec{e}_{a}\right)-\rho\left(\vec{x}-2 \vec{e}_{a}\right)\right]\right\} \\
\approx & \frac{1}{3} g c_{s}^{2} \vec{e}_{a} \cdot \nabla \rho
\end{aligned}
$$

with $\sum_{a=0}^{b} \Phi_{a}=0 . g$ is a parameter to adjust the intensity of attraction. In Eq. (6) the terms of the density difference between next nearest neighbors have been included as well as those between nearest neighbors in order to eliminate the wave-number dependence of the sound speed. Then the corresponding nonequilibrium distribution function becomes

$$
f_{a}^{*}(\vec{x}, t)=f_{a}(\vec{x}, t)+\Phi_{a} .
$$

Define

$$
\begin{gathered}
\rho^{*} \equiv \sum_{a=0}^{b} f_{a}^{*}=\sum_{a=0}^{b} f_{a}+\sum_{a=0}^{b} \Phi_{a}=\rho, \\
\rho^{*} \vec{u}^{*} \equiv \sum_{a=1}^{b} \vec{e}_{a} f_{a}^{*}=\sum_{a=1}^{b} \vec{e}_{a} f_{a}+\sum_{a=1}^{b} \vec{e}_{a} \Phi_{a} \approx \rho \vec{u}+g c_{s}^{2} \nabla \rho .
\end{gathered}
$$

Similar to the multiple phase and component model of the LB method presented by Xiaowen Shan and Hudong Chen $[16,17]$ with the attractive force, the collision does not conserve the net momentum at each site. However, it can be shown directly that the total momentum of the system ob-

TABLE III. Mach number comparison at different $u_{0}$ when $c_{s}$ $=0.52, g=0.99, \tau=1, A=0.1, T=1350, c_{s}^{\prime}=0.0556$.

\begin{tabular}{lcccccccc}
\hline \hline$u_{0}$ & 0.08 & 0.12 & 0.16 & 0.20 & 0.22 & 0.24 & 0.26 & 0.28 \\
$M^{\prime}=u_{0} / c_{s}^{\prime}$ & 1.44 & 2.16 & 2.88 & 3.60 & 3.96 & 4.32 & 4.68 & 5.03 \\
$M^{*}=1 / \sin \theta$ & 1.42 & 2.20 & 2.89 & 3.63 & 4.10 & 4.35 & 4.65 & 5.0 \\
\hline \hline
\end{tabular}

FIG. 1. The Doppler effect. 
TABLE IV. Effective sound speed comparison at different $T$ when $g=0.99, \tau=1, A=0.1, c_{s}=0.52$, $c_{s}^{*}=0.0556$.

\begin{tabular}{lccccccccc}
\hline \hline$T$ & 1750 & 1650 & 1550 & 1450 & 1350 & 1250 & 1150 & 1050 & 950 \\
$c_{s}^{\prime}$ & 0.0554 & 0.0540 & 0.0555 & 0.0545 & 0.0556 & 0.0568 & 0.0565 & 0.0576 & 0.0558 \\
\hline \hline
\end{tabular}

tained by summing over the net momentum at every site is still exactly conserved, provided no net momentum exchange has occurred at the boundary.

Having the attractive force defined, the new equilibrium function becomes

$$
\begin{gathered}
f_{a}^{* e q}=\frac{\rho}{3}\left[c_{s}^{2}+\vec{e}_{a} \cdot \vec{u}^{*}+2\left(\vec{e}_{a} \cdot \vec{u}^{*}\right)^{2}-\frac{1}{2}\left(u^{*}\right)^{2}\right], \\
f_{0}^{* e q}=\rho\left[1-2 c_{s}^{2}-\left(u^{*}\right)^{2}\right],
\end{gathered}
$$

and the kinetic equation becomes

$$
f_{a}\left(\vec{x}+\vec{e}_{a}, t+1\right)=f_{a}(\vec{x}, t)+\Phi_{a}+\Omega_{a}^{*},
$$

where

$$
\Omega_{a}^{*}=-\frac{1}{\tau}\left[f_{a}^{*}(\vec{x}, t)-f_{a}^{* e q}(\vec{x}, t)\right]
$$

It is obvious that $\Sigma_{a} \Omega_{a}^{*}=0, \Sigma_{a} \vec{e}_{a} \Omega_{a}^{*}=0$, which means $\Omega_{a}^{*}$ satisfies the conservation of total mass and total momentum at each lattice site with the attractive force.

Multiplying Eq. (11) by 1 and $\vec{e}_{a}$ and summing over $a$, after substituting Eqs. (6) and (10) and some straightforward algebra, we obtain

$$
\begin{gathered}
\frac{\partial \rho}{\partial t}+\nabla \cdot(\rho \vec{u})=0, \\
\rho\left[\partial_{t} \vec{u}+(\vec{u} \cdot \nabla) \vec{u}\right]=-c_{s}^{* 2} \nabla \rho+\nabla^{2}(\eta \vec{u})+\nabla[\nabla \cdot(\zeta \vec{u})]
\end{gathered}
$$

at low-frequency and long-wavelength limit and the effective sound speed $c_{s}^{*}$ is softened,

$$
c_{s}^{*}=\sqrt{1-g} c_{s}
$$

By Eq. (14) the critical value of $g$ is 1 , beyond which the system becomes unstable.

\section{SIMULATIONS AND RESULTS}

If we choose $\tau=1$, the kinetic equation (11) simply becomes

$$
f_{a}\left(\vec{x}+\vec{e}_{a}, t+1\right)=f_{a}^{* e q} .
$$

The advantage of Eq. (15) is that the possibility of negative distribution functions is reduced greatly.

In a rectangle field fluid flows from left to right at a uniform horizontal speed $u_{0}$. At the time $t=0$ each site has the same density $\rho_{0}(=1)$ and velocity $u_{0}$. Then we introduce a density vibration $\rho=\rho_{0}+A \sin (2 \pi t / T)$ at a single fixed site near the middle of the field. The density and speed at four boundaries remain unchanged at $\rho_{0}(=1)$ and $u_{0}$, respectively. The simulated sound speed $c_{s}^{\prime}$ is measured by the propagation speed of vibration at $u_{0}=0$. The comparison of $c_{s}^{\prime}$ and the theoretical softened sound speed $c_{s}^{*}$, which satisfies Eq. (14) is shown in Tables I and II. In those tables the value of $T$ is preselected to agree with the low-frequency and long-wavelength limit. When $u_{0} \neq 0$, the simulated Mach number is defined as $M^{\prime}=u_{0} / c_{s}^{\prime}$. The Doppler effect as $M^{\prime}<1$ and the Mach cone as $M^{\prime}>1$ are shown at different $u_{0}$; we defined the Mach number as $M^{\prime}=u_{0} / c_{s}^{\prime}$ in Figs. 1 and 2 , respectively. The theoretical value of the Mach number is calculated by the formula $M^{*}=1 / \sin \theta$, whose $\theta$ is measured in Fig. 2. The comparison of $M^{\prime}$ and $M^{*}$ is shown in Table III. Table IV shows here that the sound wave obtained is nondispersive as in reality.

\section{CONCLUSION}

In this paper, we have presented a LB model that has the capability of simulating compressible flows by introducing an attractive force. The fundamental feature of this model is the introduction of the attractive force, which effectively softens the sound speed and the Mach number is raised remarkably. Being a simple and easy-going procedure is another advantage of this scheme. Our simulation results demonstrate that this scheme can simulate compressible fluid flows with high Mach numbers up to 5. The present model is isothermal; its application is limited. However, based on this model, an extension to nonisothermal situations is expected for general applications, which is being included in our further research.

Moreover, sound speed reduction reminds us of the liquid-gas phase transition problem which should be treated by LB models with full thermodynamics. Preliminary work on a LB simulation of a van der Waals phase transition was done by us [18] with the concept of a chemical potential introduced into the model from outside. We have noticed that McNamara et al. have presented a fully correct thermal LB model; however, the numerical stability leaves much to be desired [19]. We think that the study of the phenomenon of sound speed reduction itself is of interest in connection with the problem of phase transition.

\section{ACKNOWLEDGMENTS}

We are very grateful to Dr. Hudong Chen and Dr. Yuehong Qian for instructive discussions and also wish to thank Professor Mengfen Xia for his valuable ideas and suggestions. All simulations in this paper were performed using the resources of the CFD Group at Peking University. We also thank Professor Yaosong Chen and his students for their technical support in connection with computer usage. 
[1] U. Frisch, B. Hasslacher, and Y. Pomeau, Phys. Rev. Lett. 56, 1505 (1986).

[2] Proceedings of Los Alamos Workshop, 1989, edited by G. Doolen, [Physica D 47 (1991)].

[3] B. Boghosian and D. Levermore, Complex Syst. 1, 17 (1987).

[4] D. Rothman, J. Geophys. Res. 95, 8663 (1990).

[5] H. D. Chen and W. Matthaeus, Phys. Rev. Lett. 58, 1235 (1987).

[6] S. Zaleski, The Proceedings of the Workshop on Cellular Automata and Modeling of Complex Physical Systems, Les Houches, France, 1989 (Springer-Verlag, Berlin, 1989).

[7] M. R. Swift, W. R. Osborn, and J. M. Yeomans, Phys. Rev. Lett. 75, 830 (1995).

[8] Do. Martinez, W. H. Matthaeus, S. Chen, D. C. Montgomery, Phys. Fluids 6, 1285 (1994).

[9] H. W. Liepmann and A. Roshko, Elements of Gas Dynamics (Wiley, New York, 1957).

[10] G. A. Bird, Molecular Gas Dynamics (Oxford University
Press, Oxford, 1976).

[11] F. J. Alexander, H. Chen, S. Chen, and G. D. Doolen, Phys. Rev. A 46, 1967 (1992).

[12] S. Chen, H. Chen, and G. D. Doolen, Physica D 47, 97 (1991).

[13] Y. H. Qian and S. A. Orzag, Europhys. Lett. 21, 255 (1993).

[14] Y. H. Li, L. S. Kang, and Z. J. Wu, Neural Parallel Sci. Comput. 1, 43 (1993).

[15] H. Cornille, in Proceedings of the Euromesh Colloquium 267 Series on Advanced Mathematics for Applied Science, edited by A. S. Alves (World Scientific, Singapore, 1991).

[16] Xiaowen Shan and Hudong Chen, Phys. Rev. E 47, 1815 (1993).

[17] Xiaowen Shan and Hudong Chen, Phys. Rev. E 49, 2941 (1994).

[18] Gang He and Kaihua Zhao, Commun. Theor. Phys. 29, 623 (1998).

[19] G. R. McNamara, A. L. Garcia, and B. J. Alder, J. Stat. Phys. 87, 1111 (1997). 